\title{
ANÁLISE DA QUALIDADE DA PRIMEIRA BARRA LINGOTADA NO INÍCIO DO SEQUENCIAL NA ACIARIA DA VSB - JECEABA*
}

Sávia Cristina Lacerda Poubel Pinheiro ${ }^{1}$ Luís Cláudio de Abreu Germano ${ }^{2}$ Rafael Coura Giacomin ${ }^{3}$

Takeshi Fujit

\begin{abstract}
Resumo
A cada sequencial iniciado, as primeiras toneladas de aços que caem no distribuidor podem ser contaminadas pelos refratários, bem como pelo oxigênio do ar. $\mathrm{Na}$ Aciaria da Vallourec Soluções Tubulares do Brasil (VSB), em Jeceaba, desde o start up tem-se como prática o sucateamento de aproximadamente 17 toneladas de aço no início de lingotamento, com o objetivo de minimizar problemas de qualidade no tubo devido fornecimento de barras com baixa qualidade. Neste contexto, estudos foram realizados para avaliar a qualidade destas primeiras barras de primeira corrida do sequencial, com foco em barras de diâmetro de $310 \mathrm{~mm}$. A avaliação compreendeu a análise do teor de oxigênio total ao longo da primeira barra e qualidade de tubos laminados provenientes das mesmas. Os resultados indicaram que parte da primeira barra apresenta boa qualidade para ser aplicada na laminação de tubos, reduzindo o sucateamento de barras de início de lingotamento em $7 \mathrm{t}$.
\end{abstract}

Palavras-chave: Início de lingotamento; Análise de qualidade; Inclusão.

\section{QUALITY ANALYSIS OF THE FIRST BAR CAST IN THE VSB STEELMAKING - JECEABA}

\begin{abstract}
In each new casting sequence, the first steel that comes into the tundish can be contaminated by refractory, as well as oxygen coming from the air that can react with alloys and lead to inclusion formation. In the Vallourec Soluções Tubulares do Brasil (VSB) steelmaking in Jeceaba, since the start up approximately 17 tons of steel at the casting start was scrapped as standard practice in order to minimize quality problems in the pipe due low quality. In this context, studies were carried out to evaluate the quality of this first bar from the first heat of casting sequence, focusing on $310 \mathrm{~mm}$ diameter bars. The evaluation included total oxygen content analysis along the first bar and the quality of pipes rolled from those bars. The results indicated that part of the first bar presents good quality and can be applied in the rolling mill, reducing scrapping in $7 \mathrm{t}$.

Keywords: Casting start; Quality analysis; Inclusion.

1 Engenheira de Materiais, MSc, Engenheira de Controle de Qualidade, Controle de Qualidade da Aciaria, Vallourec Soluções Tubulares do Brasil, Jeceaba, MG e Brasil.

2 Engenheiro de Produção, Coordenador Técnico, Controle de Qualidade da Aciaria, Vallourec Soluções Tubulares do Brasil, Jeceaba, MG e Brasil.

3 Engenheiro Químico, MSc em Engenharia dos Materiais. Doutorando em Engenharia dos Materiais, Materials Science and Engineering - Carnegie Mellon University. Pittsburgh - PA, EUA.
\end{abstract}


4 Engenheiro Metalurgista, MSc, Gerente de Controle de Qualidade, Controle de Qualidade da Aciaria, Vallourec Soluções Tubulares do Brasil, Jeceaba, MG e Brasil. 


\section{INTRODUÇÃO}

A Vallourec Soluções Tubulares do Brasil (VSB) é uma joint venture formada pelo grupo francês Vallourec e japonês Nippon Steel \& Sumitomo Metal Corporation (NSSMC). O complexo siderúrgico da VSB está instalado nos municípios de Belo Horizonte e Jeceaba. Os tubos produzidos pela VSB possuem alto valor agregado, atendendo à demanda do setor petrolífero mundial.

A demanda por aços de alta qualidade cresce constantemente e aplicações de determinados aços requerem baixos teores de impurezas. Neste sentido, o controle inclusional dos aços tem sido cada vez mais requisitado para fazer frente a aplicações mais severas, como exemplo em ambientes corrosivos.

A alta limpidez dos aços pode ser considerada como baixo teor de inclusões não metálicas. O conhecimento da origem química, morfologia, tamanho e distribuição das inclusões é muito importante tanto para subsidiar análises de controle no processo produtivo dos aços, quanto na avaliação de seus possíveis impactos na aplicação do produto final(1-2).

As fontes de inclusões não metálicas podem ser: (i) produto de desoxidação, onde são geradas pela reação entre o oxigênio dissolvido e o desoxidante adicionado durante o vazamento, como exemplo o alumínio; (ii) produtos de reoxidação, tais como alumina, gerada pelo alumínio remanescente no aço líquido reagindo com óxidos presentes na escória, revestimento refratário ou por exposição ao ar atmosférico; (iii) passagem de escória durante processos como vazamento; (iv) inclusões exógenas como exemplo de origem de arraste de revestimento refratário e; (v) reações químicas, como exemplo tratamento de CaSi não adequado(2).

Uma das fontes de inclusões não metálicas pode ser o início de lingotamento do aço, onde a contaminação do aço líquido no distribuidor pode estar associada à reoxidação, envolvendo interações do aço líquido, escória, revestimento refratário e/ou ar atmosférico ou passagem de escória da panela. A reoxidação do aço líquido pelo ar atmosférico pode ser considerada a principal causa de contaminação do aço no distribuidor ${ }^{(3-5)}$. Existem alguns métodos para prevenção desse processo de reoxidação no distribuidor, como a utilização de tubo longo para proteger o aço líquido durante a passagem da panela para o distribuidor, uso de pó de cobertura no distribuidor e inertização enquanto este está recebendo as primeiras toneladas de aço líquido ${ }^{(3)}$.

A aciaria da VSB instalada no município de Jeceaba possui um forno elétrico a arco, estações de metalurgia secundária sendo um forno panela e um desgaseificador a vácuo e lingotamento contínuo de barras (diâmetros de barras em 270 mm, $310 \mathrm{~mm}$ e $406 \mathrm{~mm}$ ). A máquina de lingotamento possui 5 veios e utiliza-se distribuidor de $40 \mathrm{t}$.

A cada sequencial iniciado, as primeiras toneladas de aços que caem no distribuidor podem ser contaminadas pelos refratários, bem como pelo oxigênio do ar. Dessa forma, em Jeceaba tem-se como prática o sucateamento de aproximadamente 17 toneladas de aço no início de lingotamento, com o objetivo de minimizar problemas de qualidade no tubo devido fornecimento de barras com baixa qualidade. Essa foi uma definição de projeto da Aciaria, que iniciou operação em 2011. Esforços têm sido direcionados continuamente buscando melhorar a competitividade da planta. Posto isto, a análise do sucateamento das 17 primeiras toneladas de aço lingotado se fez necessária visando avaliar a qualidade destas primeiras barras de primeira corrida do sequencial. Diante do exposto, este trabalho visa apresentar os resultados de tais análises com foco em barras de diâmetro de $310 \mathrm{~mm}$. 


\section{MATERIAIS E MÉTODOS}

Os estudos foram realizados com intuito de conhecer a qualidade da primeira barra lingotada, em termos de limpidez. As análises realizadas são descritas a seguir.

\subsection{Oxigênio Total em barra}

O oxigênio total em aço é a soma de oxigênio dissolvido e oxigênio combinado, como inclusões não metálicas. Amostras de aços no distribuidor podem ser retiradas para avaliar a limpidez da corrida.

Zhang e Thomas ${ }^{(2)}$ apresentaram este requisito como exemplo utilizado pela Kawasaki Steel, tendo como limite máximo 30 ppm de oxigênio total para amostras de aço no distribuidor para aprovação da corrida sem restrição. Para valores maiores, a aprovação é realizada após análise crítica da produção.

Para avaliar o oxigênio total neste estudo, foram retirados discos ao longo de uma barra de início de lingotamento, conforme apresentado esquematicamente na Figura 1. Além disso, foi retirado um disco no início da segunda barra de lingotamento (que é normalmente aplicada para laminação), para efeito comparativo.

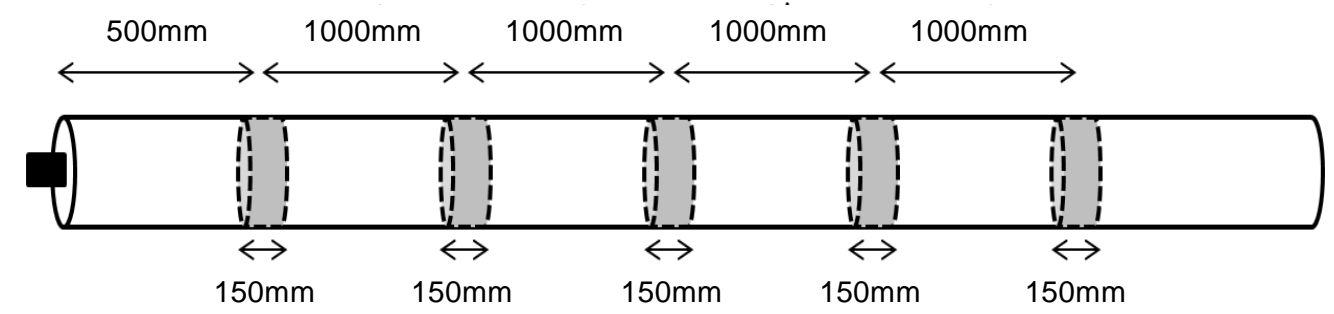

Figura 1. Desenho esquemático para retirada de discos para análise de oxigênio total em barras.

Em cada disco retirado ao longo da barra foram cortadas amostras para análise de oxigênio total. A preparação dessas amostras e o ensaio foram executados conforme critérios estabelecidos pela norma ASTM E1019: 2011 - método $\mathrm{B}^{(6)}$.

Para análise do teor de oxigênio total foi utilizado o equipamento para determinação de gases, modelo ONH2000, fabricante Eltra.

\subsection{Laminação de primeiras barras}

As primeiras barras de primeira corrida de sequencial, normalmente sucatadas na aciaria, foram laminadas experimentalmente, para conhecer a qualidade dos tubos produzidos oriundos de tais barras.

Cada barra foi cortada em 2 blocos de aproximadamente 3 metros cada, apresentados esquematicamente na Figura 2. Após laminados, cada bloco de 3 metros gerou 2 tubos (Figura 2). 
Sucata início de lingotamento $(1 \mathrm{~m})$
Bloco 1

$(3 \mathrm{~m})$
Bloco 2

$(3 \mathrm{~m})$

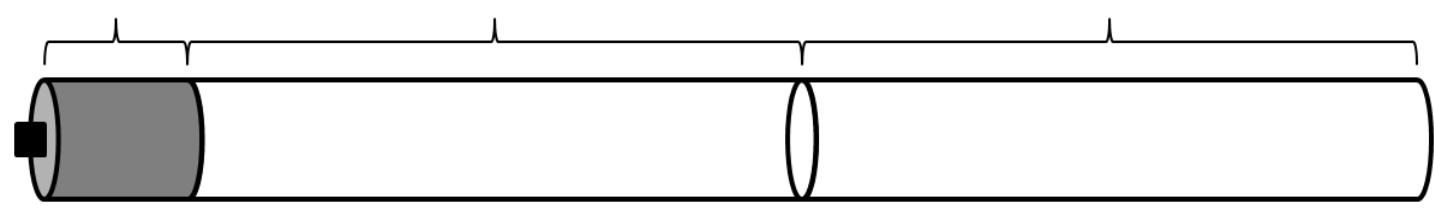

Figura 2. Esquema de divisão das primeiras barras a serem laminadas.

\subsubsection{Inspeção não destrutiva}

Cada tubo foi inspecionado em ultrassom, analisando a presença de inclusões ao longo do tubo.

\subsubsection{Ensaio de limpidez}

Para avaliar a limpidez dos tubos, amostras foram cortadas $(600 \mathrm{~mm}$ de comprimento) e realizado teste com ultrassom de bancada com vistas a determinar a quantidade de inclusões em um determinado volume de aço.

Para saber a quantidade de amostras necessárias para obter uma boa análise estatística, usou-se o cálculo de poder de teste no software Minitab ${ }^{\circledR}$, objetivando-se um poder maior que $70 \%(0,70)$ e capacidade de reconhecimento de diferenças da ordem de $50 \mathrm{~mm} / \mathrm{L}$. A Tabela 1 e Figura 3 apresentam o resultado desse cálculo baseado em uma amostragem piloto de 10 amostras.

Tabela 1. Quantidade de amostras para diferentes poderes.

\begin{tabular}{c|c|c}
\hline $\begin{array}{c}\text { Diferença entre os } \\
\text { valores (mm/L) }\end{array}$ & Poder de teste & Número de amostras \\
\hline 50 & $80 \%$ & 31 \\
\hline 50 & $90 \%$ & 41 \\
\hline 50 & $95 \%$ & 50 \\
\hline 50 & $99 \%$ & 70 \\
\hline
\end{tabular}

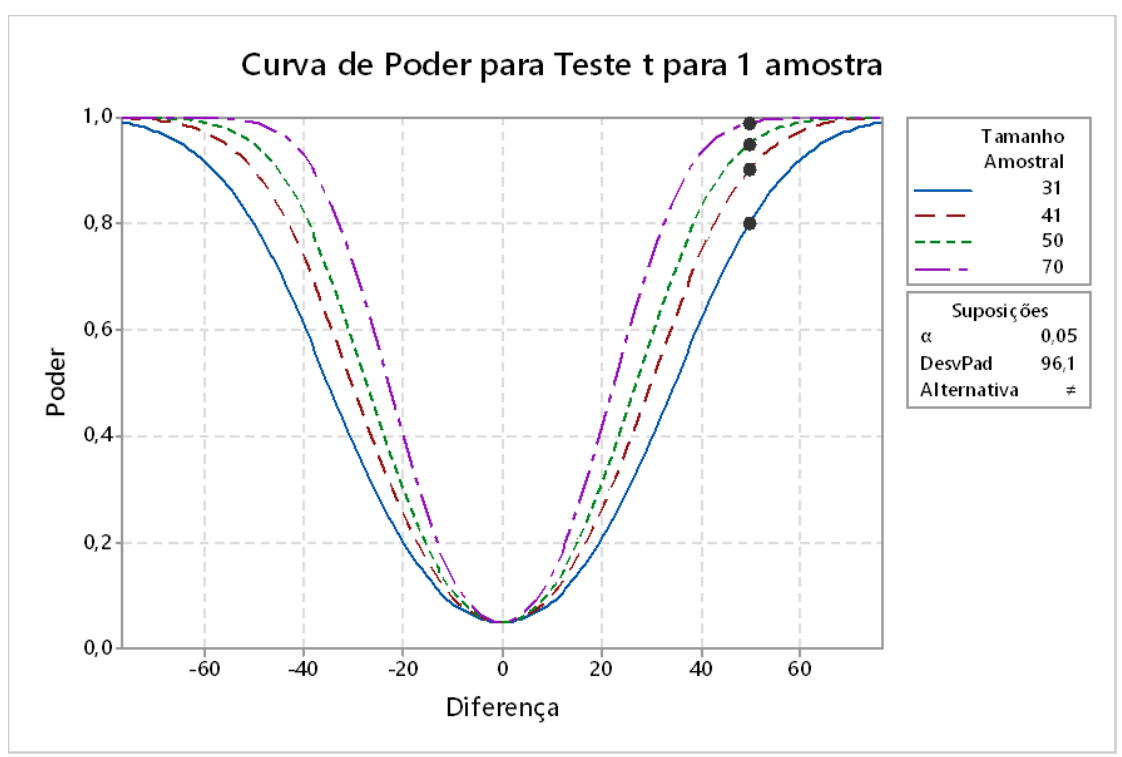

Figura 3. Quantidade de amostras para diferentes poderes. 
Os resultados demonstram que para maior poder do teste (confiança no mesmo) deve-se trabalhar com um número de amostras entre 50 e 70.16 barras foram separadas para laminar e retornaram 64 tubos laminados para avaliar a qualidade dos mesmos.

A amostragem dos tubos foi feita de modo a "varrer" desde o primeiro metro da barra (logo após a apara inicial - em cinza na Figura 2), até o final do segundo bloco. Na Figura 4 é apresentada esquematicamente a posição nas barras das amostras retiradas nos tubos.

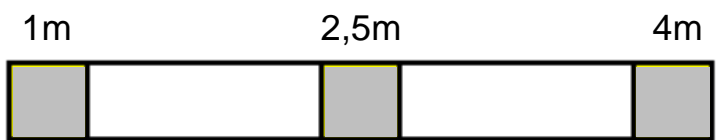

Bloco 1

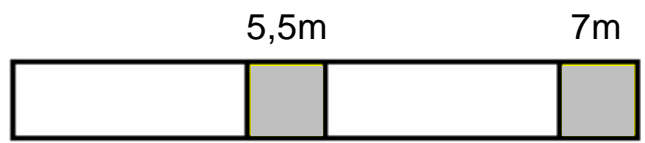

Bloco 2

Figura 4. Desenho esquemático da posição nas barras das amostras retiradas nos tubos.

Além disso, foi retirada uma amostra em cada tubo para análise de microlimpidez. A preparação das amostras e o ensaio foram executados conforme critérios estabelecidos pela norma ASTM E45, utilizando o método $A^{(7)}$.

\section{RESULTADOS E DISCUSSÃO}

\subsection{Oxigênio Total em barra}

O resultado das análises de oxigênio total ao longo da primeira barra de partida de sequencial é apresentado na Figura 5 , bem como a amostra retirada no início da segunda barra (Barra 02).

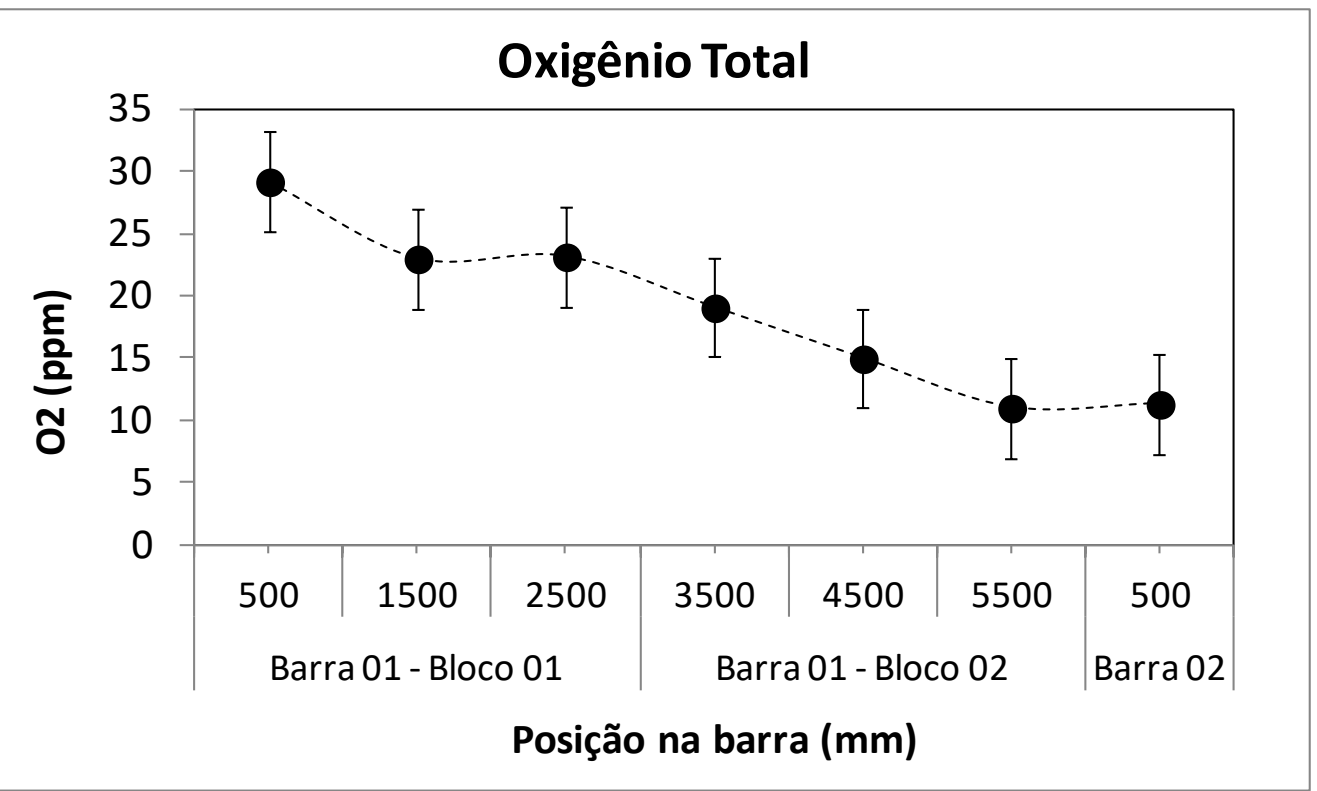

Figura 5. Resultados de oxigênio total ao longo da primeira barra de partida de sequencial.

Como esperado, observa-se uma queda nos resultados de oxigênio total ao longo da barra. O maior valor de oxigênio total encontrado foi na primeira amostra, correspondente a posição de $500 \mathrm{~mm}$ do bloco 01 da primeira barra, próximo ao cesto de selagem, sendo 29,2 ppm \pm 4 ppm (incerteza de medição da análise). 
O controle de qualidade da aciaria de Jeceaba trabalha com valor limite de oxigênio total em barras de 20 ppm para aprovação da corrida para laminar, sem necessidade de sucata adicional em barras que representem 0 início de lingotamento. Esse valor foi definido com base no histórico de análises e desempenho na laminação em termos de detecção de inclusões em tubos, durante ensaio de inspeção não destrutiva. Dessa forma, os valores encontrados na análise ao longo da primeira barra, que é dividida em dois blocos para laminar, indicaram boa qualidade para o segundo bloco da Barra 01, com valores abaixo do limite atual para aprovação de corridas.

\subsection{Laminação de primeiras barras}

\subsubsection{Inspeção não destrutiva}

O resultado do ensaio não destrutivo foi avaliado através de gráficos da função delaminação, que permite checar se há descontinuidades no meio da parede do tubo, ou seja, possíveis inclusões. Foi observada uma tendência maior de indicações de descontinuidades em tubos provenientes do bloco 1, sendo esperado, pois os primeiros metros da barra são oriundos do aço que entrou em contato com o distribuidor vazio e com o ar (antes de se jogar o pó de cobertura), além do resultado maior de oxigênio total das amostras retiradas neste bloco. Esse contato com o ar pode promover a oxidação dos elementos de liga do aço e formação de inclusões, bem como o contato com o refratário do distribuidor pode fazer com que o aço carregue partículas não metálicas para o molde.

Tubos inspecionados provenientes do primeiro bloco da primeira barra apresentaram $55 \%$ de rejeição por inclusão. Já para os tubos oriundos do segundo bloco, essas rejeições foram menores (9\%).

As indicações de descontinuidades foram amostradas e analisadas com ultrassom manual para se identificar o local exato das mesmas. Posteriormente, as amostras foram cortadas e preparadas para análise utilizando microscópio eletrônico de varredura (MEV). Em geral, o mapeamento da descontinuidade mostrou que a inclusão apresenta óxidos de alumínio, silício, titânio e traços de magnésio e cálcio (Figura 6).
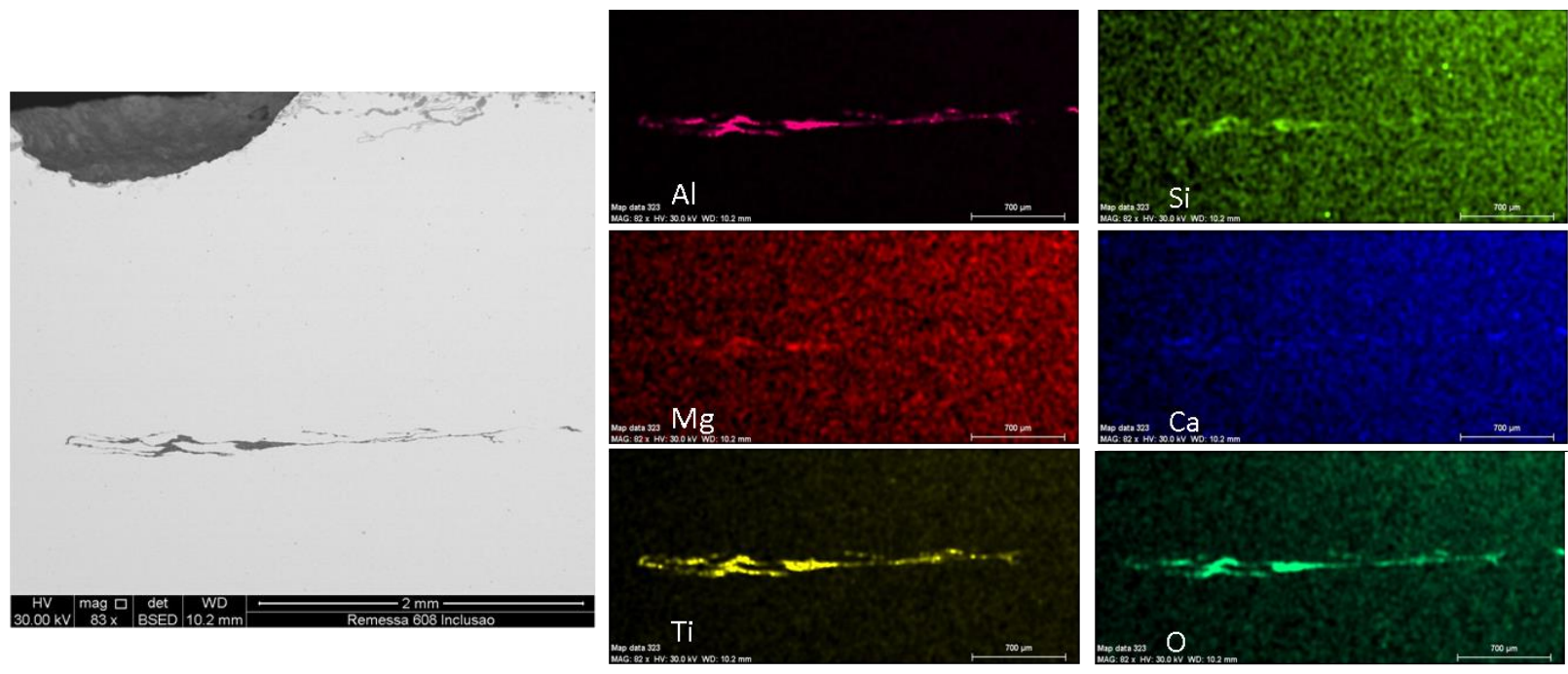

Figura 6. Fotomicrografia e resultados de mapeamento por raios-X de $\mathrm{Al}, \mathrm{Si}, \mathrm{Mg}, \mathrm{Ca}, \mathrm{Ti}$ e O da inclusão. 
A presença de titânio na inclusão indica uma possível reoxidação do aço pelo contato com ar quando da abertura da panela e distribuidor vazio. Esse tipo de inclusão foi o mesmo observado nos demais defeitos amostrados.

\subsubsection{Ensaio de limpidez}

Na Figura 7 são apresentados os resultados de ensaio de limpidez. Estes foram analisados de acordo com a posição da amostra na barra (em metros). Os valores tendem a abaixar ao longo da primeira barra.

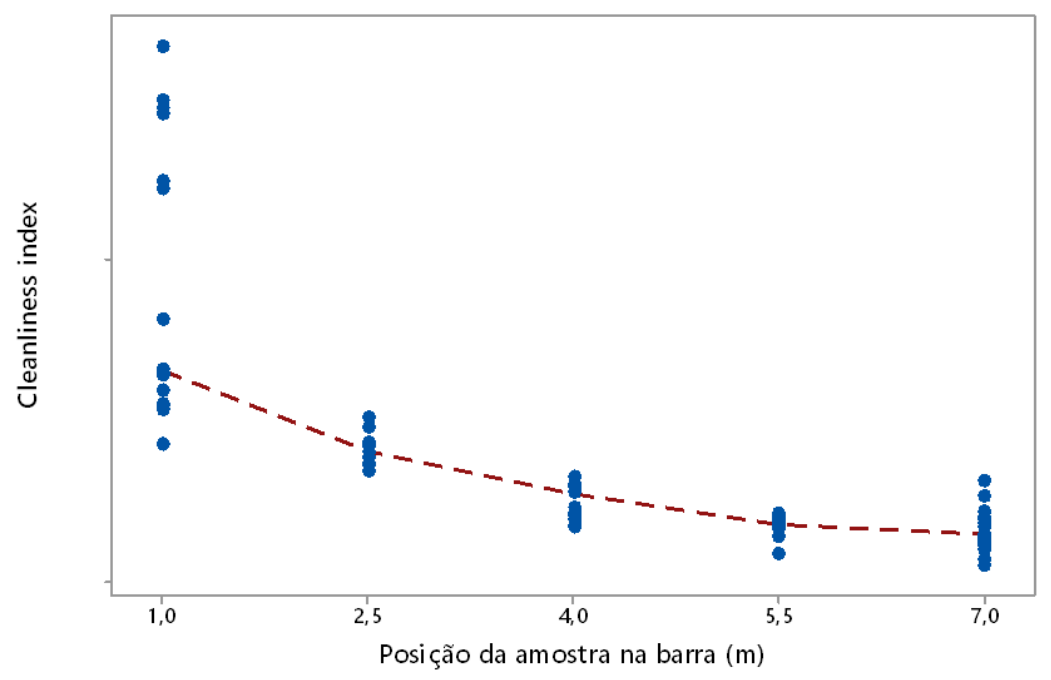

Figura 7. Resultado de limpidez por posição da amostra na barra.

Para efeito de comparação, as amostras retiradas da posição de 1 metro até 4 metros foram consideradas como constituintes do bloco 1 , enquanto amostras retiradas da posição de 5,5 e 7 metros foram consideradas constituintes do bloco 2 . A Figura 8 apresenta resultados comparativos para os blocos 1 e 2, sendo que 0 bloco 2 apresentou menor quantidade de inclusões por volume de aço analisado.

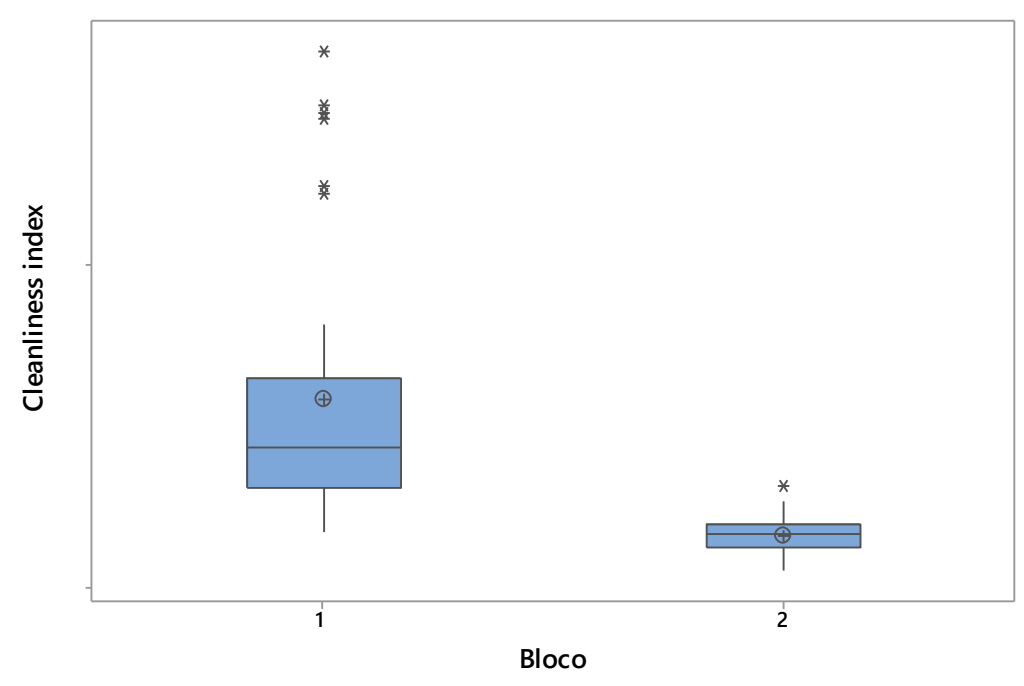

Figura 8. Resultado de limpidez por bloco. 
Para avaliar a microlimpidez dos materiais, foram retiradas amostras em todos os tubos para comparar os resultados de qualidade. Os resultados foram comparados para tubos provenientes dos blocos 1 e 2, sendo avaliado o parâmetro $B$, série fina, conforme ASTM E45, referente a inclusões de $\mathrm{Al}_{2} \mathrm{O}_{3}$ - mais importante para aços desoxidados ao alumínio.

Os valores de micropureza encontrados para ambos os blocos são valores considerados bons, porém percebe-se que os valores para o bloco 2 são menores que para o bloco 1, o que reafirma a maior limpidez do mesmo. Aproximadamente $82 \%$ das amostras apresentaram nível de severidade até 1,0 para tubos do bloco 2 e cerca de $65 \%$ deste mesmo nível para amostras do bloco 1.

Levando-se em consideração os resultados desse trabalho, foi indicado continuar o sucateamento do primeiro bloco da primeira barra lingotada no sequencial. Os resultados de qualidade para o segundo bloco indicaram que este poderia ser aplicado. Dessa forma, a redução de sucateamento por início de lingotamento seria $7 \mathrm{t}$. Essa redução foi aplicada na rotina de produção para aços sem aplicação sour service.

Adicionalmente, os resultados de qualidade de tubos laminados em termos de rejeição por inclusão são acompanhados na rotina. A taxa de rejeição de tubos por este defeito não apresentou alteração com aplicação do segundo bloco (em 2015 a taxa foi de $0,26 \%$ e em 2016 foi 0,23\%). Em paralelo, estudos vêm sendo conduzidos com objetivo de melhorar a qualidade do aço de início de lingotamento.

\section{CONCLUSÃO}

Os resultados de análise da qualidade da primeira barra lingotada no sequencial indicaram que parte desta apresenta boa qualidade para ser aplicada na laminação de tubos sem aplicação sour service. Dessa forma, a redução de sucateamento por início de lingotamento seria $7 \mathrm{t}$. Adicionalmente, a qualidade de tubos laminados tem sido acompanhada na rotina e após redução do descarte de início de lingotamento não foi observado aumento da taxa de rejeição.

\section{REFERÊNCIAS}

1 L. E. K. HOLAPPA, A. S. HELLE. Inclusion control in high-performance steels. Journal of Materials Processing Technology. 1995; 53: 177-186.

2 L. ZHANG, B. G. THOMAS. State of the art in evaluation and control of steel cleanliness. ISIJ International. 2003; 43 (3): 271-291.

3 Z. DAYA, Y. YANG, I. D. SOMMERVILLE, A. MCLEAN. Liquid steel transfer - the delivery of quality. Canadian Metallurgical Quartely. 1999; 38 (5): 285-293.

4 K. SASAI, Y. MIZUKAMI. Reoxidation behavior of molten steel in tundish. ISIJ International. 2000; 40 (1): 40-47.

$5 \mathrm{~K}$. SASAI, A. MATSUZAWA. Influence of steel grade on oxidation rate of molten steel in tundish. ISIJ International. 2012; 52 (5): 831-840.

6 ASTM E1019, 2011, STANDARD TEST METHODS FOR DETERMINATION OF CARBON, SULFUR, NITROGEN, AND OXYGEN IN STEEL, IRON, NICKEL, AND COBALT ALLOYS BY VARIOUS COMBUSTION AND FUSION TECHNIQUES, ASTM Internacional.

7 ASTM E45, 2013, STANDARD TEST METHODS FOR DETERMINING THE INCLUSION CONTENT OF STEEL, ASTM Internacional. 